\title{
Interatividade e Relações Com o Saber: Um Olhar à Luz da Análise de Redes Sociais
}

\author{
Ana Maria Casnati ${ }^{1}$, Claudia Ribeiro Santos Lopes ${ }^{2}$, Dante Galeffi ${ }^{3}$, Teresinha \\ Fróes Burnham ${ }^{3}$, Alba Benemérita Alves Vilela ${ }^{2}$, Hernane Borges de Barros \\ Pereira $^{4,5}$ \\ ${ }^{1}$ Projeto Flor de Ceibo da Universidad de La República - Uruguai \\ ${ }^{2}$ Universidade Estadual do Sudoeste da Bahia (UESB) - Campus de Jequié, BA, Brasil \\ ${ }^{3}$ Universidade Federal da Bahia, Salvador, BA, Brasil \\ ${ }^{4}$ Universidade do Estado da Bahia (UNEB), Salvador, BA, Brasil \\ ${ }^{5}$ Programa de Modelagem Computacional, SENAI Cimatec, Salvador, Bahia, Brasil \\ \{anacasnati, clopesuesb, teresinhafroes, albavilela, \\ hbopereira\}@gmail.com, dgaleffi@uol.com.br
}

\begin{abstract}
The objective of this study is to identify teachers and students social networks of the Flor de Ceibo (FDC) Program at the University de la Republica at Uruguay. The investigation applies social and complex theory observing fourteen groups (re) of FDC. Therefore, an exploratory study was carried out to demonstrate the cooperation and collaboration between teachers and students contributing to improve knowledge and social information. The findings demonstrated that teachers are no so important for the cooperation network structure. Specially the students "referents" provides effective results by improving participation and flexibility. They are very important figures for the cooperation networks and entrepreneurs who integrate other colleagues in order to prevent barriers to learning and to the synergistic profile of FDC.
\end{abstract}

Resumo. Este artigo objetiva estudar as redes sociais constituídas por professores e estudantes do projeto Flor de Ceibo (FDC) da Universidade de la Republica do Uruguai. O estudo foi desenvolvido a partir da teoria das redes sociais e complexas. Para análise foram gerados quatorze grupos (redes) que representam os territórios onde está sendo desenvolvido o FDC. As redes analisadas retratam a existência de colaboração entre professores e estudantes, resultando na interatividade ocorrida entre os atores de forma a contribuir para a difusão de informação e troca de saberes. Percebe-se que os professores não desempenham um papel central para o fluxo de interatividade sendo necessária a participação dos estudantes, e principalmente do estudante referente, para o alcance dos objetivos do FDC que considera importante a colaboração entre professores e estudantes e o uso da mediação telemática como espaços inter/transdisciplinares de formação, pesquisa, intervenção e reflexão.

\section{Introdução}

O presente trabalho é a continuação de uma pesquisa sobre redes no projeto Flor de Ceibo (FDC) da Universidade de la República, no Uruguai. Em 2012 estudando o fenômeno da interatividade em algumas redes de comunicados do projeto, Casnati, 
Lopes e Pereira concluem que o uso da teoria das redes sociais e complexas pode contribuir para compreender a interatividade nas redes educacionais, de forma a identificar os atores mais proeminentes da rede bem como servir como base para o planejamento de ações que possam levar a uma interatividade mais intensa [Casnati, Lopes e Pereira, 2012]. O estudo se complementa com uma análise do fluxo de informação nas redes de professores do Projeto Flor de Ceibo [Casnati, Lopes, Rodrigues e Pereira, 2012]. Os resultados concluem que os grupos de professores mostram uma processualidade caracterizada pelos fluxos de informação que por sua vez é responsável por transformações contínuas do projeto e da própria práxis docente.

Em consequência desses estudos, compreende-se que a escola, e também a Universidade, deve encarar novos desafios culturais e educativos decorrentes dos encontros entre ideologias, culturas, informações, imagens e difusão de produtos culturais. Atualmente o objetivo prioritário em educação é o desenvolvimento econômico e social e requer um maior nível de formação da população. Para isto, a educação deve possibilitar a resolução de problemas que derivam do seu processo de democratização.

Segundo Charlot (2007) reconhece-se nesta situação uma nova relação com o saber: cada vez mais estudantes que vão à escola querem apenas "passar de ano", sem encontrar nela sentido nem prazer. Em muitos estudantes instala-se uma postura do saber que concorda com a possibilidade de ter sucesso na escola. Em outras palavras, os estudantes não conseguem passar da situação de não-saber à condição do saber virtual ou real. Esta relação epistêmica está encarnada no corpo do aprendiz engajado no mundo.

Nesse sentido, no projeto FDC, se vivencia que o desafio é mais profundo: a interdependência crescente entre os sujeitos, a compreensão e a equidade se dá também por meio da mediação telemática. FDC é um programa da Universidade de la Republica (UdelaR) (Uy) que contribui para a formação dos estudantes comprometidos com a realidade do Uruguai e colabora com o desenvolvimento da política de democratização das Tecnologias de Informação e Comunicação (TIC), gerando espaços inter/transdisciplinares de formação, pesquisa e intervenção. Cada professor orienta um grupo com até vinte e três estudantes de diferentes cursos para trabalhar com mediação telemática em escolas e comunidades, aproveitando a experiência para gerar "espaços de reflexão" [Casnati, Lopes, Rodrigues e Pereira, 2012].

Para Charlot (2000) a análise da relação com o saber deve ser feita nas dimensões epistêmica e identitária. É nesse sentido que se pode dizer que a relação estabelecida por um sujeito com o saber é uma forma de relação com o mundo, com ele mesmo e com os outros. Com isso, a relação entre estudantes, professor e as aplicações tecnológicas informacionais de caráter conversacional migram da ideia de interação como conceito neutro a um significado conversacional de interatividade que pode contribuir e potencializar as relações com o saber.

Na mesma direção, surge a concepção da mediação telemática como dispositivo intermediário de tradução que assegura o diálogo e que corresponde à noção de interface. Assim, Silva (2010) distingue uma interatividade tecnológica "onde prevalece a comunicação e a troca de mensagens" e a interatividade situacional "definida como possibilidade de agir/interferir no programa e/ou conteúdo" o que também contribui na relação com o saber.

Diante deste cenário, o presente artigo tem por objetivo estudar as redes sociais constituídas por professores e estudantes do FDC para analisar os fluxos de 
comunicação/interação e suas interrelações com o saber. Neste panorama, a Teoria das Redes Sociais contribui para a análise de fluxos de interatividade em ambientes multirreferenciais de aprendizagem [Casnati e Galeffi, 2014] de forma a identificar os atores mais proeminentes da rede e servem como base para a planificação de ações que possam levar a uma coordenação e melhores propostas pedagógicas em relação aos interesses dos estudantes, adequados aos contextos culturais de atuação dos grupos do FDC.

\section{Método de análise}

O estudo foi desenvolvido a partir da Teoria de Análise de Redes Sociais e Complexas. As redes analisadas são resultantes das interações (mensagens) ocorridas entre professores e estudantes do projeto FDC, obtidas por meio da Plataforma EVA (Moodle), o qual está disponível com licença GPL (Licença Pública Geral).

O FDC trata-se de uma proposta educativa universitária com encontros presenciais semanais e comunicações virtuais. Cada grupo está conformado por um docente e 15 estudantes. O professor relaciona-se com seus estudantes e, por sua vez, os estudantes de cada grupo relacionam-se entre si nos fóruns de cada grupo no ambiente de aprendizagem EVA do Flor de Ceibo. Para tanto, cada grupo tem um espaço especifico para comunicação, criação coletiva, gestão de usuários, aprendizagem colaborativa e ferramentas de avaliação e essas atividades são desenvolvidas no ambiente Moodle/EVA.

Dessa forma, foi possível extrair os dados para análise a partir dos fóruns de comunicados de cada grupo, considerando a mensagem enviada por um estudante A ao estudante B, bem como a resposta emitida pelo estudante B ao estudante A, além das mensagens emitidas pelo Professor responsável. O professor responsável pelo grupo também pode propor um tema no fórum da plataforma EVA ou na sessão presencial e depois os estudantes respondem no ambiente virtual, provocando uma interatividade entre aqueles que integram o grupo.

Como ferramenta utilizada para a extração e tabulação dos dados foi utilizada a planilha eletrônica MS Excel. Os vértices das redes representam os professores e estudantes (atores) enquanto que as arestas as mensagens trocadas entre eles. Todas as turmas foram monitoradas durante os meses de maio a julho de 2011.

No presente trabalho foi gerado quatorze grupos (redes) os quais representam os territórios onde esta sendo desenvolvido o FDC. Para caracterizar as redes utilizaram-se parâmetros das redes complexas, e, dentre esses se destacam nesse estudo o número de vértices $(n=|\mathrm{V}|)$, arestas $(m=|\varepsilon|)$, grau médio $(\langle k\rangle)$, caminho mínimo médio $(L)$, diâmetro da rede $(D)$, densidade $(\Delta)$ e coeficiente de aglomeração médio $(C)$ [Freeman, 1978].

O número de vértices de uma rede representa os atores que a compõe enquanto que as arestas as relações existentes entre os vértices. Uma aresta, nas redes estudadas, ocorria entre vértices $A$ e $B$ quando um vértice $A$ enviava uma mensagem para um vértice $B$, bem como a mensagem enviada de um vértice $B$ para um vértice $A$. Dois vértices são ditos adjacentes se são os extremos de uma aresta $a$ de um grafo [Lucchesi et al., 1979], [Szwarcfiter, 1984]. O grau médio $(\langle k\rangle)$ de uma rede representa o número médio de arestas incidentes nos vértices de uma rede

Chama-se de caminho mínimo entre os vértices ou distância, ao menor caminho existente entre dois vértices [Lucchesi et al., 1979], [Szwarcfiter, 1984]. O diâmetro de uma rede, entretanto, equivale ao maior menor caminho entre dois vértices, e informa o 
quão afastados estão na rede. A densidade $(\Delta)$ é uma métrica que leva em conta o número e a proporção das arestas, sendo uma medida de coesão da rede. Ou seja, quanto mais arestas uma rede tiver, mais densa a rede será.

A medida da densidade, para esta pesquisa, tem um significado teórico especial, pois, as redes densas sugerem a existência de um fluxo máximo de interatividade para a relação com o saber entre os atores uma vez que a densidade depende do número de conexões existentes dividido pelo número de conexões possíveis. Quanto maior a densidade da rede, maior a interação e fluxo de saberes na rede. Um grafo completo tem todas as arestas possíveis e a densidade igual a 1.

O coeficiente de aglomeração de um vértice $i$ é uma medida de densidade da rede formada pela vizinhança do vértice, ou seja, pelos vértices que se ligam ao vértice $i$. Quanto maior for a quantidade de ligações entre os vizinhos de $i$, maior será a sua aglomeração. Logo, o coeficiente de aglomeração médio $(C)$ da rede representa a média do coeficiente de aglomeração de seus vértices [Barabasi e Albert, 2003].

Para estudar os dados, além de métricas das redes complexas, utilizaram-se também índices para análise de redes sociais. Dentre esses, se destacam nesse estudo a centralidade de grau $\left(C_{g}\right)$, de intermediação $\left(C_{i}\right)$ e de proximidade $\left(C_{p}\right)$ [Freeman, 1978], [Wasserman e Faust, 1994]

O conceito de centralidade de grau se relaciona com o número de arestas que um ator possui com outros atores na rede, indicando a centralidade local do vértice. A centralidade de proximidade está inversamente relacionada à distância entre os vértices de uma rede. Logo, é possível concluir que quanto menor for a distância de um vértice com o restante da rede, maior será a sua centralidade de proximidade.

A centralidade de intermediação, por sua vez, permite avaliar as interações entre dois vértices não adjacentes a partir dos vértices que se localizam no caminho entre eles. Dessa forma, para que um vértice possua uma alta centralidade de intermediação é necessário que o mesmo esteja no caminho entre diversos outros vértices. Esse índice possibilita identificar os vértices importantes para a distribuição de informações que circulam na rede e contribuem para uma maior conectividade entre os atores. No caso do projeto FDC acredita-se que os estudantes desempenham um importante papel para difusão de saberes com seus pares em outros serviços universitários.

\section{Análise e discussão dos resultados}

As redes analisadas são constituídas por um número de vértices que oscila entre 4 e 24 e com um número de arestas variando entre 7 e 153. Isso indica que a conformação dos grupos por território no projeto FDC é heterogênea, apresentando características diversas.

Essa diversidade pode ser percebida a partir do: número de vértices, o qual retrata como o grupo é formado; número de arestas, que mostram as interações que ocorrem na rede; grau médio $(\langle k\rangle)$, que indica o número médio de conexões existentes entre os vértices na rede (variando entre 3,0 a 7,29), sendo o grau de entrada indicativo das mensagens recebidas, enquanto que o grau de saída o número de mensagens enviadas pelo vértice; diâmetro $(D)$, variando entre 2 e 6 , o qual equivale à maior distância entre os vértices na rede; densidade $(\Delta)$, variando entre 0,12 e 0,64 , sendo a rede que apresenta $\Delta=0,12$ a de menor conectividade entre os atores e aquela com $\Delta=0,64$ a que apresenta uma maior conectividade entre os atores da rede, e essa conectividade reflete no fluxo de interatividade e saberes da rede; coeficiente de aglomeração médio 
(C), com valores variando entre 0,17 e 0,73 , sendo a rede com $C=0,73$ a que apresenta o maior fluxo de interatividade e troca de saberes entre os vértices na rede. No Quadro 1 é possível observar as características gerais das redes analisadas.

\section{Quadro 1: Características das redes por território que integram o projeto Flor de Ceibo - Universidade de La República de Uruguay}

\begin{tabular}{|c|c|c|c|c|c|c|c|}
\hline Rede & $\mathbf{N}^{\circ}$ Vértices & $\mathbf{N}^{\circ}$ Arestas & $\boldsymbol{D}$ & $\boldsymbol{L}$ & $\Delta$ & $\boldsymbol{C}$ & $\langle\mathbf{K}\rangle$ \\
\hline 1 & 12 & 28 & 5 & 2,44 & 0,21 & 0,21 & 5,66 \\
\hline 2 & 9 & 15 & 5 & 2,44 & 0,20 & 0,17 & 3,55 \\
\hline 3 & 19 & 84 & 6 & 2,18 & 0,24 & 0,56 & 5,21 \\
\hline 4 & 17 & 46 & 4 & 2,35 & 0,16 & 0,47 & 3,05 \\
\hline 5 & 4 & 7 & 2 & 1,41 & 0,58 & 0,54 & 3,00 \\
\hline 6 & 18 & 42 & 3 & 2,05 & 0,12 & 0,46 & 4,10 \\
\hline 7 & 15 & 60 & 4 & 1,85 & 0,15 & 0,42 & 6,45 \\
\hline 8 & 24 & 153 & 4 & 1,91 & 0,28 & 0,46 & 7,29 \\
\hline 9 & 11 & 62 & 2 & 1,47 & 0,56 & 0,66 & 6,27 \\
\hline 10 & 14 & 76 & 3 & 1,61 & 0,41 & 0,63 & 6,92 \\
\hline 11 & 10 & 49 & 3 & 1,49 & 0,54 & 0,68 & 7,20 \\
\hline 12 & 8 & 36 & 2 & 1,35 & 0,64 & 0,73 & 6,37 \\
\hline 13 & 8 & 20 & 2 & 1,59 & 0,35 & 0,47 & 3,62 \\
\hline 14 & 11 & 49 & 3 & 1,78 & 0,44 & 0,53 & 5,00 \\
\hline
\end{tabular}

Fonte: os autores

Apesar dessa heterogeneidade, dentre as redes resultantes neste estudo observase que na sua maioria são redes esparsas (medianamente conectadas), entretanto destacam-se as redes 11 e 12 entre aquelas que apresentaram um coeficiente de aglomeração $(C)$ acima de 0,5 que equivale ao valor médio dos coeficientes de aglomeração obtidos com as redes analisadas.

Além disso, quanto ao diâmetro das redes analisadas, percebe-se que algumas, no caso as redes 1 e 2 , apresentaram um valor de $D=5$ indicando que os vértices encontram-se mais distantes na rede, ou seja, há um menor fluxo de interatividade entre os atores que as compõem. Essas redes também apresentaram os menores valores de coeficiente de aglomeração $(C=0,21$ e $C=0,17$, respectivamente), característica essa que vem reforçar a ocorrência de um menor fluxo de interatividade entre os atores, percebida a partir do diâmetro apresentado, quando comparado às demais redes analisadas.

Diante desses resultados, para fins de visualização e análise do comportamento das redes, serão consideradas as redes de número $1,2,11$ e 12 , uma vez que as mesmas apresentaram os extremos da realidade observada, tendo as redes 1 e 2 o índice $C<0,5$ enquanto que as redes 11 e 12 o índice $C>0,5$ conforme pode ser observado no Quadro 1. O coeficiente de aglomeração $(C)$ indica o quanto uma rede se encontra conectada. Uma rede pode ser considerada completamente conectada quando possui $C=1$.

\subsection{Redes que apresentaram menor fluxo de interatividade $(C<0,5)$}

As redes 1 e 2 foram as que apresentaram menor fluxo de interatividade, com valores de $C=0,21$ e $C=0,17$, respectivamente. Com isso, observa-se que esses são os grupos do projeto FDC que possuem conexões mais esparsas, indicando pouca interatividade entre os atores. Para verificar os vértices que apresentavam maior fluxo de interatividade na rede foram observados os índices de centralidade de grau $\left(C_{g}\right)$, de intermediação $\left(C_{i}\right)$ e de proximidade $\left(C_{p}\right)$.

Na rede 1 é observado que o professor (vértice P36) não é o ator central uma vez que os valores de centralidade desse vértice $\left(C_{g}=6 ; C_{i}=18\right.$; e $\left.C_{p}=2,09\right)$ são menores 
quando comparados aos apresentados pelo estudante referente _ vértice $\mathrm{AE} 1\left(C_{g}=11\right.$; $C_{i}=53$; e $\left.C_{p}=1,63\right)$.

Nesse sentido, o estudante referente se apresenta com uma maior importância para o fluxo de interatividade dessa rede na relação com o saber. O estudante referente tanto recebe quanto transmite as informações e discussões dos colegas e do professor para outros atores que compõem a rede, de forma a contribuir para intensificar a interatividade entre os atores. Os valores encontrados equivalentes às centralidades de cada ator na rede são apresentados no Quadro 2.

Quadro 2: Centralidades de Grau $\left(C_{g}\right)$, de Intermediação $\left(C_{i}\right)$, de Proximidade $\left(C_{p}\right)$ dos vértices na rede Grupo 1

\begin{tabular}{|c|c|c|c|c|c|}
\hline Vértice & Grau de Entrada & Grau de Saída & $\boldsymbol{C}_{\boldsymbol{g}}$ & $\boldsymbol{C}_{\boldsymbol{i}}$ & $\boldsymbol{C}_{\boldsymbol{p}}$ \\
\hline $\mathrm{AE} 1$ & 5 & 6 & 11 & 53,0 & 1,63 \\
\hline $\mathrm{AE} 2$ & 4 & 2 & 6 & 14,5 & 2,36 \\
\hline $\mathrm{AE} 3$ & 3 & 2 & 5 & 10,0 & 2,45 \\
\hline $\mathrm{AE} 4$ & 2 & 6 & 8 & 14,0 & 1,63 \\
\hline P36 & 4 & 2 & 6 & 18,0 & 2,09 \\
\hline AE5 & 2 & 2 & 4 & 10,0 & 2,36 \\
\hline AE6 & 2 & 2 & 4 & 19,5 & 2,36 \\
\hline AE7 & 1 & 1 & 2 & 0,5 & 3,18 \\
\hline AE8 & 1 & 1 & 2 & 13,5 & 2,36 \\
\hline AE9 & 2 & 2 & 4 & 28,5 & 2,63 \\
\hline AE10 & 1 & 1 & 2 & 4,0 & 3,0 \\
\hline AE11 & 1 & 1 & 2 & 5,5 & 3,27 \\
\hline
\end{tabular}

Tanto o estudante referente quanto o professor apresentam conexões estabelecidas entre esses com os demais atores na rede e possuem menor distância com relação aos vértices mais distantes na rede. Dessa forma, destaca-se a importância deles para a difusão de informações e troca de saberes na rede.

É possível perceber ainda que existe protagonismo e colaboração nas ações do grupo uma vez que todos os atores tem recebido e enviado informação, ou seja, tem interagido na rede (Figura 1). Porém alguns vértices se destacam com menor fluxo de interação, como é o caso dos vértices AE7, AE10 e AE11.

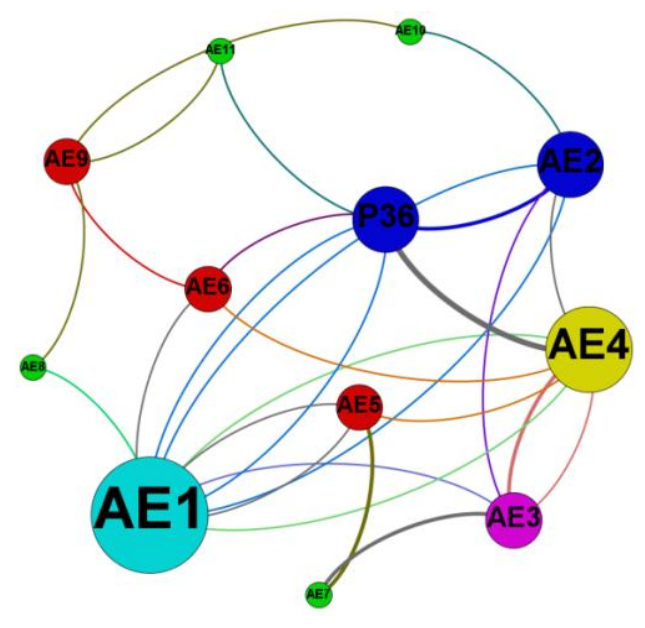

Figura 1: Rede Grupo 1 (Fonte: Os autores)

Isso nos leva à necessidade de planejamento e gerenciamento de ações que promovam uma maior conectividade e troca de saberes na rede de forma a atingir plenamente os objetivos do projeto FDC. Tais ações devem ser protagonizadas pelo 
professor responsável sem desconsiderar a importante participação dos estudantes e, em especial, do estudante referente.

Na rede 2 (Figura 2, Quadro 3) o coeficiente de aglomeração $(0,18)$ em relação à rede $1(0,22)$ é menor e isso revela uma menor atividade da rede, característica esta que se confirma quando são comparadas as densidades de ambas as redes: o valor da densidade da rede 2 é 0,17 e na rede 1 é 0,21 .

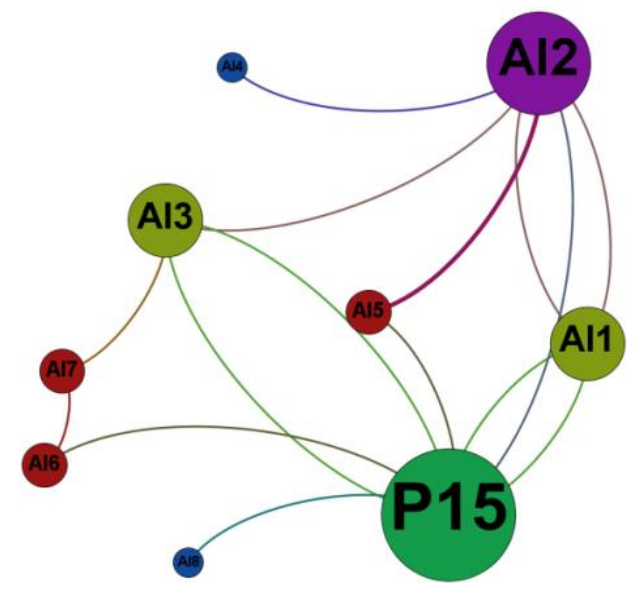

Figura 2: Rede Grupo 2 (Fonte - Os autores)

O vértice professor se apresenta com maior importância para a interatividade que ocorre na rede e ocupa uma posição central no fluxo comunicacional e na relação com o saber. Essa importância está caracterizada pelos valores apresentados quanto as centralidade de grau $\left(C_{g}=8\right)$ e de intermediação $\left(C_{i}=29,5\right)$.

O professor mais recebe do que envia mensagens na rede, o que evidencia certa centralização de informação. Entretanto, o inverso acontece com relação ao estudante referente (AE1) uma vez que envia mais mensagens do que recebe. Esse comportamento ressalta, mais uma vez, a importância do estudante referente para intensificar a interatividade entre os atores na rede.

Quadro 3: Centralidades de Grau $\left(C_{g}\right)$, de Intermediação $\left(C_{i}\right)$, de Proximidade $\left(C_{p}\right)$ dos vértices na rede Grupo 2

\begin{tabular}{|c|c|c|c|c|c|}
\hline Vértice & Grau de Entrada & Grau de Saída & $\boldsymbol{C}_{\boldsymbol{g}}$ & $\boldsymbol{C}_{\boldsymbol{i}}$ & $\boldsymbol{C}_{\boldsymbol{p}}$ \\
\hline $\mathrm{P} 15$ & 6 & 2 & 8 & 2,14 & 29,5 \\
\hline $\mathrm{A} 11$ & 2 & 2 & 4 & 2,14 & 17,0 \\
\hline $\mathrm{A} 12$ & 1 & 5 & 6 & 14,29 & 14,5 \\
\hline $\mathrm{A} I 3$ & 2 & 2 & 4 & 2,42 & 11,0 \\
\hline $\mathrm{A} 14$ & 1 & 0 & 1 & 0,0 & 0,0 \\
\hline $\mathrm{A} 15$ & 1 & 1 & 2 & 2,71 & 0,0 \\
\hline $\mathrm{A} 16$ & 1 & 1 & 2 & 2,71 & 6,0 \\
\hline $\mathrm{A} I 7$ & 1 & 1 & 2 & 3,28 & 6,0 \\
\hline $\mathrm{A} 18$ & 0 & 1 & 1 & 2,87 & 0,0 \\
\hline
\end{tabular}

Fonte - Os autores

Assim como na rede 1, na rede 2 tanto o professor quanto o estudante referente se apresentam com maior importância para a difusão de informações e troca de saberes na rede. Se percebe ainda a existência de um protagonismo e colaboração nas ações dos vértices que compõem a rede 2 , ocorrendo intercâmbio de mensagens entre todos os atores. Apenas o vértice AI4 não colaborou com as discussões da rede por ter abandonado o programa. 


\subsection{Redes que apresentaram maior fluxo de interatividade $(C>0,5)$}

As redes 11 e 12 apresentaram maior fluxo de interatividade dentre os grupos analisados, tendo $C>0,5$ (coeficiente de aglomeração da rede). A rede 11 é constituída por dez atores e a rede 12 por oito atores.

$\mathrm{Na}$ rede 11 os vértices que apresentaram as maiores centralidades de grau e intermediação foram P20 que representa o professor $\left(C_{g}=16\right.$ e $\left.C_{i}=2,25\right)$ seguido de VA2 que representa o estudante referente $\left(C_{g}=14\right.$ e $\left.C_{i}=9,53\right)$. Nesse sentido, é observado que esses vértices tanto recebem quanto transmitem mensagens na rede, de forma a contribuir para o fluxo de interatividade entre estudantes e professor.

Nesta rede (Figura 3, Quadro 4) há um fluxo mais intenso na troca de mensagens, com uma participação de todos os atores. O professor (vértice P20) se conecta diretamente com todos os estudantes, e com isso desempenha um importante papel na difusão de informações e interatividade da rede, porém de forma não centralizadora, o que coincide com a filosofia do FDC onde os estudantes desempenham um protagonismo importante. Nesse sentido também se destaca o papel do estudante referente, que apesar de não se conectar diretamente com todos os outros vértices, também contribui de forma significativa para a troca de saberes na rede.

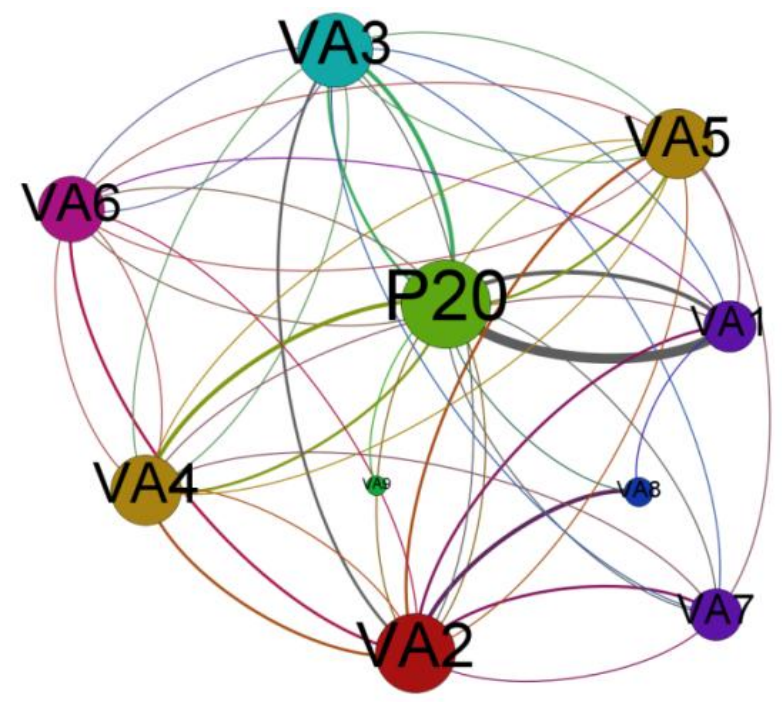

Figura 3: Rede do Grupo 11 (Fonte- Os autores)

Quadro 4: Centralidades de Grau $\left(C_{g}\right)$, Intermediação $\left(C_{i}\right)$ e Proximidade $\left(C_{p}\right)$ dos vértices na rede Grupo 11

\begin{tabular}{|c|c|c|c|c|c|}
\hline Vértice & Grau de Entrada & Grau de Saída & $\boldsymbol{C}_{\boldsymbol{g}}$ & $\boldsymbol{C}_{\boldsymbol{i}}$ & $\boldsymbol{C}_{\boldsymbol{p}}$ \\
\hline P20 & 9 & 7 & 16 & 1,12 & 2,25 \\
\hline VA1 & 7 & 1 & 8 & 2,0 & 0,0 \\
\hline VA2 & 6 & 8 & 14 & 1,0 & 9,53 \\
\hline VA3 & 6 & 7 & 13 & 1,12 & 1,53 \\
\hline VA4 & 5 & 7 & 12 & 1,12 & 0,2 \\
\hline VA5 & 5 & 7 & 12 & 1,12 & 0,2 \\
\hline VA6 & 5 & 6 & 11 & 1,25 & 0,0 \\
\hline VA7 & 5 & 3 & 8 & 1,62 & 0,0 \\
\hline VA8 & 1 & 2 & 3 & 1,75 & 0,0 \\
\hline VA9 & 0 & 1 & 1 & 2,0 & 0,0 \\
\hline
\end{tabular}


Na rede 12 (Figura 4 e Quadro 5) os vértices que mais se destacaram também são aqueles que representam o professor (P13) e o estudante referente (VIA2) do grupo, o mesmo ocorrido nas demais redes analisadas. As trocas de mensagens e saberes nesta rede foram intensos, e todos os vértices participaram ativamente. O professor contribuiu de forma significativa para isso uma vez que interagiu com todos os atores da rede.

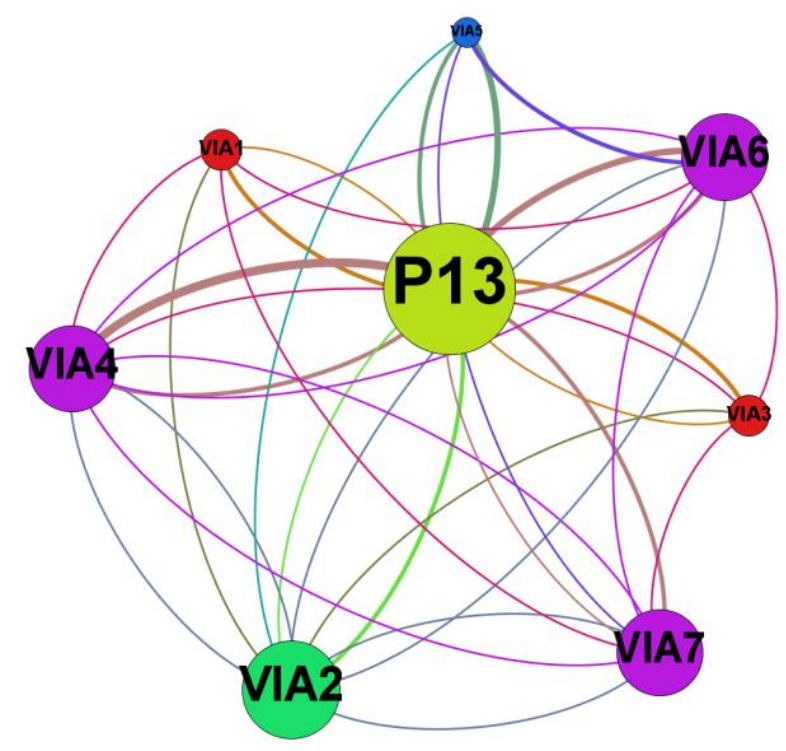

Figura 4: Rede Grupo 12 (Fonte- Os autores)

Quadro 5: Centralidades de Grau $\left(C_{g}\right)$, Intermediação $\left(C_{i}\right)$ e Proximidade $\left(C_{p}\right)$ dos vértices na rede Grupo 12

\begin{tabular}{|c|c|c|c|c|c|}
\hline Vértice & Grau de Entrada & Grau de Saída & $\boldsymbol{C}_{\boldsymbol{g}}$ & $\boldsymbol{C}_{\boldsymbol{i}}$ & $\boldsymbol{C}_{\boldsymbol{p}}$ \\
\hline P13 & 7 & 7 & 14 & 1,0 & 1,85 \\
\hline VIA1 & 5 & 1 & 6 & 1,85 & 0,0 \\
\hline VIA2 & 4 & 7 & 11 & 1,0 & 0,58 \\
\hline VIA3 & 5 & 1 & 6 & 1,85 & 0,0 \\
\hline VIA4 & 4 & 6 & 10 & 1,14 & 0,33 \\
\hline VIA5 & 4 & 1 & 5 & 1,85 & 0,0 \\
\hline VIA6 & 4 & 6 & 10 & 1,14 & 0,25 \\
\hline VIA7 & 3 & 7 & 10 & 1,0 & 0,25 \\
\hline
\end{tabular}

Fonte - Os autores

Apesar dessa participação ativa do professor, é importante destacar o papel dos estudantes para esse fluxo intenso da rede, tendo mais uma vez o estudante referente como aquele que apresentou as maiores centralidades de grau e intermediação dentre os estudantes. Esses resultados traduzem a política implementada pelo programa de FDC, a qual busca uma interatividade e troca de saberes, com a valorização do protagonismo dos estudantes.

\section{Considerações finais}

Através da análise de redes sociais comprova-se a existência de diferentes padrões de conformação dos grupos (redes) no projeto FDC, variabilidade essa condicionada ao comportamento fundamentalmente dos professores e estudantes referentes que se manifesta na variação espacial. 
A partir das características encontradas nas redes estudadas, se observa formatos de funcionamento e dinâmica do fluxo comunicacional e interatividade diferentes, porém, com comportamentos semelhantes dos atores em cada rede o qual pode ser explicado em razão da estrutura horizontal do próprio FDC. Essa horizontalidade permite um desenvolvimento mais ágil, proativo e inovador no contexto educativo uruguaio.

Nesse sentido, a rede aparece como um modelo de cooperação que contribui nas relações com o saber e intercambio de conhecimento muitas vezes deixando de ser tão dependentes da centralização e proximidade do professor e da função docente. Assim os fluxos nas relações com o saber dos integrantes definem o grau de interação na rede, interferindo diretamente na sua evolução nas relações com o conhecimento.

A troca de conhecimentos e aprendizados proporcionada pelos laços de conexão das redes estabelece e condiciona o processo dos integrantes, pois a atividade mediada telematicamente acaba redundando em formas de gestão do conhecimento. Logo, a possibilidade simbólico-analítica da análise de redes sociais contribui para a identificação de problemas nas atividades de intermediação e comunicação nos ambientes educativos contemporâneos.

A principal contribuição deste trabalho, observada a partir dos resultados encontrados, é a conclusão de que a metodologia de análise de redes permite detectar o papel fundamental que os estudantes referentes desempenham na compreensão da dinâmica do projeto. Isso fica evidenciado com a análise do comportamento dos estudantes referentes na maioria das redes, onde se pode afirmar que essas revelam um novo contexto, um novo meio de máxima pluralidade e incontornável singularização onde os eventos comunicativos de aprendizagem acontecem.

Dessa forma, tanto a pluralidade quanto a singularidade do processo depende da orientação docente e das intervenções e experiência dos estudantes referentes. A modalidade dos estudantes referentes foi inaugurada em 2010 e a metodologia de análise de redes evidencia que eles são importantes no intercâmbio e relação com os saberes.

Destaca-se que o estudante referente é uma inovação do FDC com a finalidade de manter os demais estudantes no projeto e contribuir para a sua formação trabalhando e pesquisando em mediação telemática e conhecimento, e a análise ora desenvolvida evidencia que a implantação do papel do estudante referente contribui de maneira fundamental de forma a organizar e consolidar os grupos do FDC.

Neste trabalho, o uso da análise de redes sociais como ferramenta possibilitou inferir que aprendizagem e relação com o saber é uma atividade sem lugar certo e ainda assim é poderoso. FDC é um conjunto cooperativo de professores e estudantes universitários que utilizam e aplicam a energia intelectual, linguística e a construção comunicativa para as relações com o saber e conhecimento.

Os resultados alcançados com este estudo levaram a uma posterior análise qualitativa do tipo etnográfica, e as redes analisadas constituem uma fotografia do projeto FDC em 2011, sendo necessário continuar estudando a evolução das relações identificadas no transcurso do tempo. Porém, com os resultados ora apresentados, podese concluir que a partir da Teoria das Redes Sociais é possível estabelecer um retrato da interatividade e relações com o saber uma vez que mostram a existência de colaboração entre professores e estudantes, resultando na interatividade ocorrida entre os atores, de forma a contribuir para a difusão de informação e troca de saberes. 


\section{Referências}

BARABÁSI, L.; ALBERT, G. (2003). Linked: how everything is connected to everything else and what it means for business, science, and everyday life. [S. 1.]: A Plume Book.

CASNATI, A. M.; LOPES, C. R. S.; PEREIRA, H. B. B. (2012). Interatividade em Educação: uma análise a partir das redes sociais e complexas. Anais do XI Congreso Latinoamericano de Investigadores de La Comunicación - ALAIC, 2012, realizado na cidade de Montividéu - Uruguai.

CASNATI, A. M.; LOPES, C. R. S.; RODRIGUES, A. A. A. de O.; PEREIRA, H. B. de B. (2012). Análise do Fluxo de Informação e Interatividade nas Redes de Docência do Projeto Flor de Ceibo (Uy). Proceedings of the Brazilian Workshop on Social Network Analysis and Mining - BRASNAM, XXXII Congress of the Brazilian Computer Society, Curitiba, Brazil.

CASNATI, A. M.; LOPES, C. R. S.; GALEFFI, Dante; PEREIRA, H. B. B. (2014). Interactivity in education: social and complex network analysis. Arcila, C., Calderin, M. and Castro, C. (Org.) An overview of digital media in Latin America. VISTAS University of West London Press, c/o INSPIRE, University of West London, St Mary's Road, Ealing, London, United Kingdom.

CASNATI, A.; GALEFFI, D. (2014). La interactividad em ambientes multirreferenciales de aprendizaje. Em Rev. Intercâmbios, vol.2,n ${ }^{\circ} 1$, dic. p. 49-58.

CHARLOT, B. (2000). Da relação com o saber: elementos para uma teoria. Porto Alegre: Artes Médicas Sul.

CHARLOT, B. (2007). Educação e Globalização: uma tentativa de colocar ordem no debate. Sisifo. Rev . de Ciências da Educação, n. 4, out/dez.

FREEMAN, L. C. (1978). "Centrality in social networks: Conceptual clarification". Social Networks, 1:215-239.

LUCCHESI, C. L. et al. (1979). Aspectos teóricos da computação. IMPA - Rio de Janeiro: [s.n.].

SILVA, M. (2010). Sala de aula interativa. Rio de Janeiro: Loyola.

SZWARCFITER, J. L. (1984). Grafos e algoritmos computacionais. Rio de Janeiro: [s.n.].

WASSERMAN, S. e FAUST, K. (1994). "Social network analysis: methods and applications". Cambridge University Press, Cambridge. 